\title{
Distribución comercial y marketing en el sector turístico cubano.
}

\section{Commercial distribution and marketing in the cuban tourist sector.}

\author{
Antonio Díaz Medina. ${ }^{1}$
}

\begin{abstract}
An analysis of commercial distribution and marketing in the tourist sector as factors of the decreasing tendencies of the key indicators of the Cuban touristic destination. The documents explores the peculiar Cuban situation to apply marketing tools to run the state enterprises, especially in tourism, including the marketing variable which is the focus of the present investigation, commercial distribution.
\end{abstract}

Key Words: Commercial Distribution, Marketing, Socialism.

\section{Resumen}

Un análisis de la distribución comercial y el marketing en el sector turístico cubano como factores a tener en cuenta en la tendencia decreciente de varios indicadores claves del destino Cuba. Profundiza en la situación peculiar de Cuba para la aplicación del marketing en la gestión de los sectores de la economía, en particular el turismo, incluida la variable objeto de análisis en la investigación que se lleva a cabo, la distribución comercial.

Palabras Clave: Distribución Comercial, Marketing, Socialismo.

\section{Camino recorrido en la investigación}

Se viene investigando la distribución comercial de productos (servicios) turísticos en general, desde el esclarecimiento de que es turismo, su origen y nacimiento, como abordarlo desde la ciencia, el conocimiento científico sobre él, para después pasar a los actores que intervienen en este fenómeno social.

Los resultados hasta este punto se resumen en:

\footnotetext{
${ }^{1}$ Prof. Auxiliar, Facultad de Turismo Universidad de La Habana, antonio_diaz@ftur.uh.cu
} 
- $\quad$ El turismo no puede asumirse como industria, sino como lo que es, un fenómeno social.

- $\quad$ Que este fenómeno nace, o se "inventa“ en el siglo XVIII inglés, aunque tiene antecedentes de épocas anteriores.

- $\quad$ Que una de las esencias del turismo es el motivo de ocio.

- $\quad$ Que como tal hay que estudiarlo multidisciplinariamente.

- Uno de estos enfoques es el económico y este nos lleva a las empresas que ofertan servicios asociados a la demanda de servicios que genera dicho fenómeno y a los consumidores, llamados turistas, que los demandan y consumen.

- Que estas empresas ofertan servicios de dos tipos: los básicos, que son anteriores al turismo: transporte, alojamiento, restauración, entretenimiento; y

- Que hay otras empresas que ofertan servicios de nivel superior, que llamo nivel 2. Estos servicios integran varios servicios básicos que devienen nuevos productos complejos, paquetes, circuitos, etc.

- Que estas empresas ofertan sus servicios no sólo a los turistas, sino a otros viajeros y clientes de todo tipo, con motivos disímiles, como lo hicieron siempre desde antes del advenimiento del turismo.

- Que como empresas, que ofertan en el mercado global, internacional, nacional y local, pueden estudiarse desde el marketing como disciplina. (Díaz, 2012)

El estudio desde el marketing nos lleva a cada uno de sus componentes o variables, dentro de la que priorizamos la distribución comercial, por ser el campo en que nos desarrollamos como profesionales y ahora como académicos.

Se han realizado varias investigaciones de conjunto con estudiantes, algunos de cuyos resultados, trabajos de diploma para su graduación (se refieren en cada caso concreto), se utilizan en el presente trabajo por su contribución al establecimiento de la situación de la distribución comercial del producto turístico cubano, aún sin concluir.

El objetivo final es establecer el comportamiento de cada una de las variables del marketing en el caso cubano y hasta donde este es utilizado propiamente en la gestión de los principales actores del sector.

\section{Introducción}

El turismo tiene gran impacto en la sociedad cubana, es uno de los sectores líderes de su economía (Varios). Es por tanto una necesidad conocerlo como fenómeno social y como negocio para la economía cubana.

Ha sido abordado profusamente de varias formas por la academia cubana y mundial: como fenómeno social (Díaz, 2012) Como sector de la economía también ha sido estudiado intensamente (Martin, 2006) y desde varios ángulos, tanto su derrama a otros 
sectores, como lo que significa en la creación de empleo, como destino y como comercializarlo, conservarlo, promoverlo, etc. (Thomas et al, 2015) (WTTC, 2017) (Rodríguez, 2012) (Ayala, 2014)

Desde el marketing y sus variables son muchas las investigaciones y trabajos publicados, incluidos trabajos de diploma (Varios), de maestrías (Varios) y doctorados (Martin, 2006) de nuestra Facultad y de otras facultades de la UH (Barreiro, 2013) y otras universidades del país, al estar presente el turismo en prácticamente todo el territorio nacional (Machado, Hernández, 2010); (Arnaiz, Pérez, Aspiolea, 2017), (Seguí; Rodríguez; 2012). Son también notables los estudios desde la sostenibilidad, la ecología y el clima. (Laitamaki, 2018), (Torres, 2016)

Sobre todos estos temas se realiza de una u otra forma recopilación de información que sirve de soporte para estos estudios y análisis. Un tema bien atendido, al menos desde el punto de vista de la información, es la calidad, la satisfacción del cliente, en el entendido que esto tiene un impacto decisivo en la comercialización del producto turístico cubano. (Seguí, Rodríguez; 2012) (Cuza, 2013), (López, 2013)

Sin embargo, hay un componente del marketing que ha sido estudiado relativamente poco en nuestro país. Se trata de la distribución comercial del producto turístico y de viajes. Hay estudios que tocan el tema de la participación de algunos tipos de intermediarios en la comercialización del producto cubano, pero pocos en los que ha sido la distribución comercial su objeto de estudio como tal (Rodríguez, 2017), prevaleciendo sobre todo los asociados al comercio electrónico (Pérez, 2016), salvo los tutorados por el que subscribe y otros profesores de la asignatura "Gestión de Agencias de Viajes", de la Facultad de Turismo. (Rodríguez. 2017) (Errasti, Diaz. 2016), (Rodríguez, Díaz. 2016), (Horta, Diaz, 2017)

El presente trabajo presenta algunos de los avances en la investigación sobre la distribución comercial del producto turístico cubano y su posible impacto en los resultados del sector en el presente siglo en nuestro país.

La investigación se viene desarrollando por etapas, comenzando por algunos destinos, como La Habana y Sancti Spiritus, las cadenas hoteleras, tanto extranjeras como nacionales, que deben completarse con un destino importante de sol y playa y las agencias de viajes nacionales, conocidas como Receptivos. Se proyectan estudios sobre algunos de los principales mercados emisores, en particular Canadá y Europa para intentar tener una visión integral del tema.

\section{Resultados del turismo en este siglo en Cuba.}

Si se analiza la entrada de visitantes al país, (Fig 1), se verifican variaciones significativas a la tendencia sostenida al crecimiento que hemos tenido desde que el turismo se convirtió 
en prioridad como "industria” en Cuba. (MINTUR, 2018) Algunas pueden atribuirse a problemas exógenos a la comercialización, a los que este negocio es bien sensible, como las crisis económicas, naturales o político-sociales, incluidas las guerras sobre todo de carácter internacional.

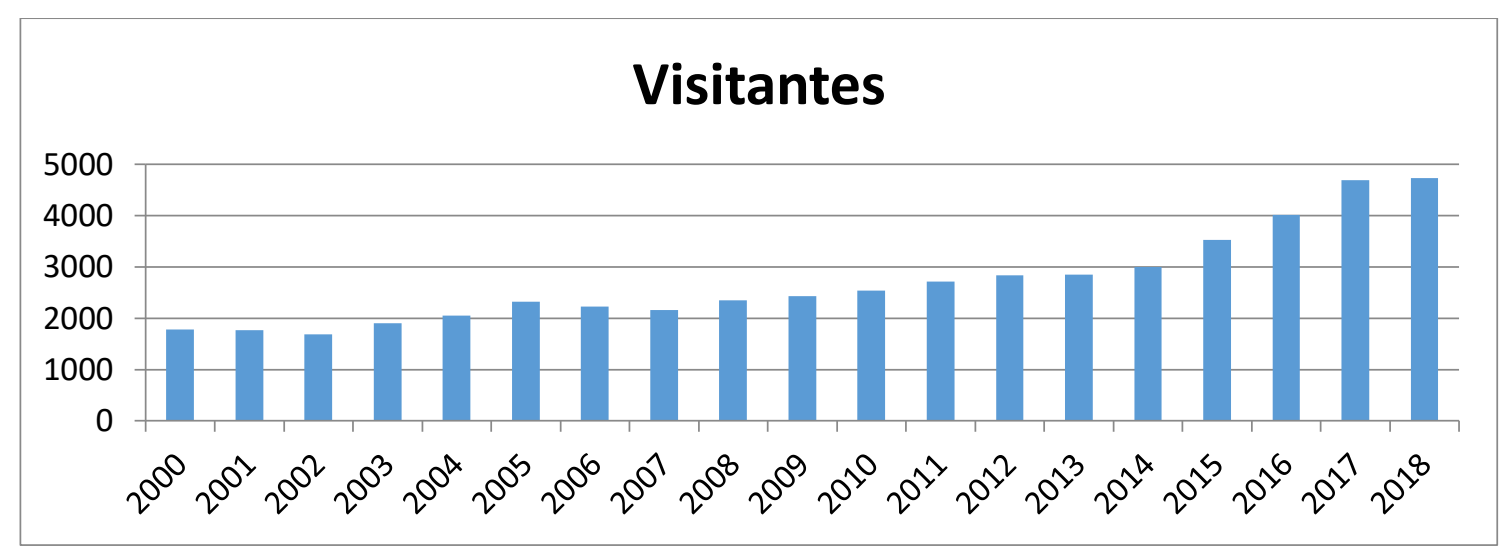

Figura 1. Visitantes.

Fuente: Mintur

Las guerras desatadas por EE.UU. contra Afganistán e Iraq en el 2001 deprimieron la industria en ese año y el siguiente en Cuba, como se ve en el gráfico, sin embargo la Gran Depresión del 2008 no mermó el crecimiento. Es significativo también la disminución del ritmo de crecimiento en la primera década del presente siglo XXI con relación a la última del XX, bien estudiada y presentada por el Dr. Figueras. (Figueras, 2013)

De 1990 a 1999 la tasa de crecimiento fue del $19 \%$, mientras del 2000 al 2011 fue sólo del $4 \%$, (Figueras, 2013) Figueras agrega otro dato aún más significativo, en la última década del siglo pasado los ingresos por turismo crecieron incluso a un ritmo superior que los arribos, a un $23 \%$ anual, pero en los primeros 10 años de la presente centuria estos han crecido por debajo del crecimiento de las entradas de clientes, a un deprimido $2 \%$.

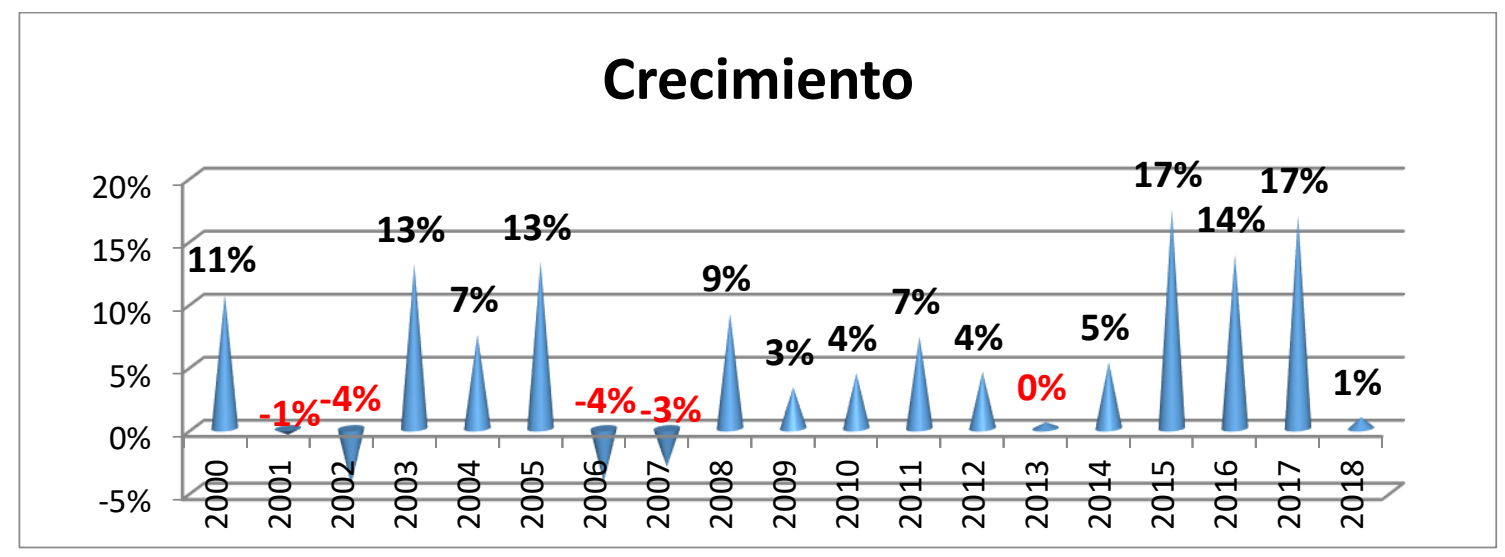

Figura 1. Crecimiento.

Fuente: Elaboración Propia 
Este autor argumenta sobre las causas de la disminución del ritmo de crecimiento de los ingresos, una de las cuales es la reducción de llegadas del mercado europeo en un $20 \%$, pero hay que encontrar el porqué de esta disminución de entradas de turistas de ese continente, el segundo mercado regional para Cuba después del norteamericano liderado por Canadá.

Hubo estancamientos en los arribos al destino en el 2006, 2007 y 2013 no asociados a factores externos.

Las causas Figueras las dividía entre externas e internas, comenzamos por las externas: (Figueras, 2013)

- El agresivo desarrollo de nuevos destinos mercados (Turquía, Marruecos, Tunez, Egipto),

- La reaparición de destinos tradicionales en la anterior antigua Yugoslavia, los destinos de Europa del Este,

- $\quad$ Encarecimiento de los pasajes aéreos al haberse triplicado el precio del jet fuel.

- $\quad$ Las causas internas según este autor fueron: (Figueras, 2013)

- Insuficiente agresividad y seguimiento sistemático a los factores decisorios en esos mercados emisores, (Promoción y estudio de mercado)

- Descenso en la calidad de los servicios, (Producto)

- Deterioro de un alto por ciento de instalaciones, (Producto)

- Concentración de las inversiones en instalaciones del sistema Todo Incluido, que facilitan aprovechar el potencial y ventajas en cuanto a calidad de las playas y el clima, pero quedando un vacío en otras opciones turísticas, (Producto)

- $\quad$ El no aprovechamiento del turismo de ciudad con múltiples ofertas culturales y de otro tipo. (Promoción, Producto)

Habría que agregar factores de distribución comercial, incluidas las variaciones en esos mercados de cómo se vende, disminución del peso de los turoperadores, incremento del papel de las OTAs que dominan prácticamente el mercado, y del producto aéreo ofertado, favoreciendo los destinos más cercanos, sin compensación para los vuelos a Cuba, de larga distancia, tanto por turoperadores como por líneas regulares. Esto es ahora más obvio, por la situación creada por la pandemia del Covid-19.

A partir de diciembre del 2014, con el restablecimiento de relaciones con EE.UU. se produjo en estos mercados europeos una tendencia al alza que se verifica también en el mercado europeo, una motivación descrita como "tratar de ver a Cuba antes de que las relaciones con EE.UU. la cambien totalmente". 
El descenso en estos 3 años solo puede ser achacado a problemas de marketing y sus 4 variables básicas, pues son años antes de la crisis más aguda del capitalismo mundial en más de 100 años que ocurrió a partir del 2008, años cuando los arribos al país subieron. Hay también estudios sobre factores externos (Valdivia, 2010) que inciden en el Turismo en Cuba, pero es necesario estudiar las razones para estos descensos que parecen ubicadas en la política de marketing aplicada en el país, algunas relacionadas con los canales de distribución como la disminución del co-marketing a los principales turoperadores en los mercados emisores más importantes posterior al 2004.

Por otra parte es crónica la tendencia decreciente del promedio de estancia del turista en Cuba (Figueras, 2013), uno de los factores de la también crónica disminución de los ingresos por turistas. (Tabla \# 1)

Tabla 1: Cuba: Ingresos del turismo, por visitante y por turista.

\begin{tabular}{|c|c|c|c|c|c|c|}
\hline CONCEPTO & 2013 & 2014 & 2015 & 2016 & 2017 & 2018 \\
\hline Visitantes & $2,862,273$ & $3,013,584$ & $3,540,175$ & $4,009,169$ & $4,653,559$ & $4,711,910$ \\
\hline Turistas & $2,838,684$ & $2,980,664$ & $3,506,106$ & $3,975,268$ & $4,593,914$ & $4,683,655$ \\
\hline Pernoctaciones & $25,612,125$ & $25,739,533$ & $29,916,768$ & $33,202,458$ & $33,085,823$ & $28,840,669$ \\
\hline Promedio estancia en Cuba & 9.0 & 8.6 & 8.5 & 8.4 & 7.2 & 6.2 \\
\hline Ingresos turismo internacional & $\$ 2,325,100,000$ & $\$ 2,367,300,000$ & $\$ 2,600,800,000$ & $\$ 2,907,100,000$ & $\$ 3,185,900,000$ & $2,903,100,000$ \\
\hline Ingresos por turista & $\$ \quad 819$ & $\$ \quad 794$ & $\$ \quad 742$ & $\$ \quad 731$ & $\$ \quad 694$ & $\$ \quad 620$ \\
\hline Ingreso por turista/dia & 91 & 92 & 87 & 88 & 96 & 101 \\
\hline
\end{tabular}

Fuente: Anuario Estadístico 2017.

Más significativa aún es la disminución sostenida de los ingresos por turista/día, devenida en parte por la disminución de los días de estancia, en esta tabla (Tabla \# 2), que llega hasta el 2017 para eliminar el efecto de las medidas que ha venido aplicando la Administración del Presidente Trump, pero que si incluya la bonanza experimentada en los años finales de su predecesor, el Presidente Obama, que no modifica la tendencia decreciente.

Tabla 2: Llegadas e ingresos turísticos. Principales receptores, Caribe Insular y Mundo.

\begin{tabular}{|c|c|c|c|c|c|c|c|c|c|c|c|c|c|}
\hline \multirow[b]{2}{*}{ Destinos } & \multicolumn{4}{|c|}{ Miles de Llegadas de turistas } & \multicolumn{4}{|c|}{ Millones USD de Ingresos turísticos } & \multicolumn{5}{|c|}{ Ingresos por Turista } \\
\hline & 2010 & 2016 & 2017 & CMA* & 2010 & 2016 & 2017 & CMA* & & 2010 & & 2016 & 2017 \\
\hline R. Dominicana & 4,125 & 5,959 & 6,188 & $4.5 \%$ & $\$ \quad 4,163$ & 6,720 & $\$ \quad 7,178$ & $8.1 \%$ & $\$$ & 1,009 & $\$$ & 1,128 & $\$ 1,160$ \\
\hline Puerto Rico & 3,186 & 3,736 & 3,797 & $2.5 \%$ & 3,211 & 3,985 & 4,090 & $3.3 \%$ & $\$$ & 1,008 & $\$$ & 1,067 & $\$ 1,077$ \\
\hline Cuba & 2,507 & 4,009 & 4,653 & $6.8 \%$ & 2,187 & 2,907 & 3,186 & $5.4 \%$ & $\$$ & 872 & $\$$ & 725 & $\$ \quad 685$ \\
\hline \begin{tabular}{|l|} 
Jamaica \\
\end{tabular} & 1,922 & 2,181 & 2,353 & $4.3 \%$ & 2,001 & 2,539 & 2,809 & $5.0 \%$ & $\$$ & 1,041 & $\$$ & 1,164 & 1,194 \\
\hline Bahamas & 1,370 & 1,484 & 1,439 & $1.0 \%$ & 2,163 & 2,591 & 2,577 & $2.2 \%$ & $\$$ & 1,579 & $\$$ & 1,746 & 1,791 \\
\hline Caribe Insular & 19,521 & 25,153 & 25,957 & $3.1 \%$ & $\$ 22,809$ & $\$ 30,036$ & $\$ \quad 31,729$ & $4.8 \%$ & $\$$ & 1,156 & $\$$ & 1,194 & 1,222 \\
\hline Mundo & 951,000 & $1,239,000$ & $1,323,000$ & $4.5 \%$ & $\$ 989,000$ & $\$ 1,245,000$ & $\$ 1,340,000$ & $5.5 \%$ & $\$$ & 1,018 & $\$$ & 1,005 & 1,013 \\
\hline
\end{tabular}

Fuente: Elaboración propia.

Los ingresos totales y por turista son también cada vez menores relativamente comparados con el del resto del Caribe y el Mundo. El ingreso por turista en Cuba es sólo un $72 \%$ del resto del Mundo y apenas un $60 \%$ del de nuestra área, el Caribe. (Tabla \# 2). Seguimos una tendencia de decrecimiento totalmente opuesta al resto de los destinos 
del área y se requiere encontrar las causas de esta notable diferencia para poder revertirlos con una renovada estrategia de marketing.

Todas estas tendencias decrecientes llegan a nuestros días aún con el crecimiento obtenido por el mal llamado "efecto Obama", que fue de dos dígitos a partir del 2016 en cuanto a visitantes, pero menor al año anterior en estancia media e ingresos por turista. (Tabla \# 1)

\section{Mercado y socialismo}

Una causa de estos resultados descendentes puede encontrarse en cómo abordamos el marketing en nuestra economía, definida como socialista, basada en la planificación. Son varios los trabajos sobre el tema en este siglo dentro de la academia cubana. (Barreiro, 2013), (Hernández; Díaz y Barreiro; 2009), (Souto y Pérez: 2012), (Díaz, Barreiro, 2006), (Díaz, Pons, 2009)

A pesar de los cambios ocurridos desde finales del pasado siglo, que apuntan a darle alguna participación al mercado, autorizando a nuevos actores, llamados cuentapropistas, que se suman a los pequeños agricultores privados y a las cooperativas en el sector agrícola, no se utiliza al mercado como instrumento de dirección económica, a pesar de lo definido en la "Resolución Económica" del V Congreso del Partido en 1998, algo que ahora se propone remediar, al menos en parte, según la nueva Constitución (Artículo 18).

Se minimiza la objetiva presencia del mercado tanto en las relaciones económicas internas como con el exterior desaprovechándose, somos de la opinión, el natural juego dialéctico planificación/mercado que en el socialismo, por primera vez como sistema económico, puede funcionar con la prevalencia del primero. (García, 2014)

Se ha profundizado sobre qué se entiende por marketing en una sociedad socialista, (Barreiro, 2003) y cómo asumirlo en el turismo, en tanto forma más acabada de dirección de entidades económicas que producen bienes o servicios de cualquier tipo, comercializados en mercados, así como para la promoción de destinos turísticos locales y nacionales, incluida Cuba.

Hay que entender el nacimiento y evolución posterior del marketing. (Shaw y Jones, 2005). La literatura habla del marketing como hecho, asociado al nacimiento y desarrollo del comercio y el marketing como objeto de estudio por la academia que comienza según estas fuentes en los albores del siglo XX y con el nombre de "Distribución" (Jones, 2009). Ya aquí comienzan los desentendidos y confusiones sobre el término y como utilizarlo. (Bartels, 1976).

Su momento de florecimiento coincide con productores, capitalistas, con productos sin salida, en la situación creada por la Gran Depresión en los años 30, (Jones, 2009) con la ampliación de la producción, nuevos productos de consumo, nuevos servicios, mayor competencia, en el mismo escenario de producción capitalista signado por las crisis de superproducción que crean la necesidad imperiosa de evitarlas. Parece que su desarrollo está asociado con la agudización de factores como: el aumento creciente de la 
productividad, de la concurrencia y la competencia, en lo que se necesita profundizar. (Borden, 1964);

Otro problema, por no decir tendencia a encubrir la esencia del sistema socio económico, es llamar a las economías capitalistas como economías de mercado (Bartels, 1976). Error, en nuestra opinión, que gran cantidad de economistas y personas de todas las avenidas arrastran hasta hoy mismo, incluyendo los que se adscriben a las ideas socialistas y al materialismo dialéctico. El resultado es realmente grave, pues primero esconde la esencia del sistema, y la relación social de producción en que se basa, el capital y el trabajo asalariado, y de rebote, convierte al mercado en su "esencia" engañosa, lo que "bloquea" artificialmente su uso por los demás sistemas que han sido y son. Esto aplica para la teoría del socialismo, aunque nos parece no tan difícil de limpiarla de este fardo apócrifo. (García, 2014)

El socialismo ha existido en un Mundo que sigue siendo mayoritariamente capitalista, al cual hay que incorporarse participando en el mercado mundial a vender lo producido y comprar lo necesario (Resolución Económica V Congreso del PCC). El mercado sigue funcionando dentro de nuestra economía (Pons, 2008), (García, 2014) y negarlo solo nos dificulta entenderlo y utilizarlo para los fines de la sociedad como una necesidad objetiva que emana del estado de las fuerzas productivas y de las relaciones de producción que aún persisten, definidas no sólo por la planificación centralizada, sino, al menos en la misma proporción, por la necesidad del mercado. (García, 2014)

\section{Marketing y comercialización del turismo en Cuba.}

El trabajo del Dr. Ramón Martín ya mencionado, analiza la condición de Oligopolio del turismo en Cuba, (Martin 2006). Esto supone que a pesar de la centralización férrea de la economía cubana, aún existe alguna variedad de oferta de productos y servicios turísticos y un nivel apreciable de competencia. Consideramos que esto ha venido cambiando hacia la concentración y las más recientes decisiones con la creación de OSDEs monopólicas, las llamadas Organizaciones Superiores de Dirección Económica, en temas como las agencias de viajes confirman esta tendencia.

A partir de mediados de la pasada década el crecimiento hotelero del país es fundamentalmente de GAVIOTA (“Excelencias Cuba“). En realidad, la poca competencia que podía haber hasta esa fecha entre la oferta controlada por el MINTUR y la del grupo GAVIOTA apenas es notable, lo que requiere ser estudiado a profundidad.

Parece aplicarse el marketing centralmente para todo el Destino Cuba. La Resolución Económica del V Congreso del PCC de 1997 ya incluía directivas para el sector del turismo que definen buena parte de su estrategia de marketing:

:Su misión como captador directo de divisas frescas, ocupando una posición competitiva en el mercado."

"Potenciará las formas actuales de comercialización e incorporará nuevas modalidades como el multidestino,". 
“La integración de los diferentes servicios y opciones, y su diversificación, contribuirán" para "mejorar la calidad del producto turístico cubano (......) y (.....) ofrecer paquetes de servicios cada vez más integrados y que logren el máximo efecto financiero neto."

Pero ¿hasta donde los demás actores: los Destinos específicos dentro del país, y sobre todo, las empresas estatales que dominan totalmente la oferta del destino, están en condiciones para aplicar estrategias y gestión de marketing al no verificarse en la práctica la independencia económica para ellas?. La investigación sobre la distribución de los hoteles de la provincia Sacnti Spiritus deja claro que esos hoteles no cuentan con independencia alguna a tales fines. (Rodríguez, Díaz. 2016)

La condición de monopolio en uno o dos únicos centros de decisiones del país para el sector del turismo pasa por los recursos que pueden utilizar los actores subordinados a dicho centro, léase destinos, clusters, empresas. Estos no disponían de fondos propios, generados por sus resultados, para su gestión de marketing, incluso para reponer parte de los medios básicos que integran sus productos, pues hasta los fondos por depreciación eran entregados al Estado hasta la aparición del Decreto Ley 320 en abril del 2014. (DECRETO-LEY Nos. 320 y 323).

Un análisis reciente sobre el llamado Perfeccionamiento Empresarial deja claro los problemas que se han confrontado en este intento:

Las concepciones y prácticas erradas sobre la realización de la propiedad social, bajo la forma estatal y la planificación central, conducen a ignorar o subestimar el rol que debe tener la gestión empresarial en cuanto al emprendimiento y la innovación, el aprovechamiento de las oportunidades del mercado, la captación y generación de recursos financieros, la necesidad de tomar decisiones bajo condiciones de autonomía y asunción de riesgos para el aprendizaje de una gestión responsable. (del Castillo, 2016, pag. 3)

Esto nos lleva a la revisión del marco legal del sistema empresarial en Cuba que ha venido renovándose desde el 2007. El Decreto Ley 252 emitido ese año con intenciones descentralizadoras incluye dentro de sus principios los límites de la "descentralización”:

"la combinación adecuada, armónica y oportuna, de las decisiones que son tomadas centralmente por el Gobierno con aquellas que se delegan al sistema empresarial". (Decreto Ley 252, 2007, pag2)

El Gobierno central toma una buena parte de las decisiones, las principales, y delega otras a las empresas. La clave es determinar cuáles delega y si estas son suficientes para que las empresas puedan aplicar el marketing en su gestión.

El 252 fue complementado por el 281 en la misma fecha que es el reglamento para su aplicación y ambos fueron modificados 5 años después por el Decreto-Ley No. 295 y el 303, dirigidos a mejorar las funciones y atribuciones del Grupo Gubernamental que controla el proceso. Un par de años después vuelven a modificarse estos Decretos de 
nuevo con modificaciones sobre las prerrogativas del órgano rector. (DECRETO-LEY Nos. 252, 281, 276 y 295).

Pero más reciente, en el año 2014 se aplicaron dos nuevos Decretos, el 320, modificando el 252 del 2007 de nuevo, incluyendo lo ya apuntado sobre la Depreciación y el 323, modificando el 281, que crea las llamadas OSDEs, Organo Superior de Dirección Empresarial, que en esencia busca "separar las funciones estatales de las empresariales" y para ello concentra en menos manos, una sola, un grupo de empresas, formando en algunos casos Monopolios como ETECSA en las telecomunicaciones, o VIAJES CUBA, el que agrupa al $80 \%$ de las agencias de viajes estatales cubanos, y en otros casos conglomerados de varios tipos de productos y servicios como los que ya existían del tipo de la Corporación CIMEX. (DECRETO-LEY Nos. 320 y 323).

En base a este escenario todo apunta a que aplicar el marketing en el sector turístico en Cuba sólo le es posible objetivamente al MINTUR y a GAVIOTA, como monopolios asociados, pues las nuevas OSDEs, las empresas y otras instituciones del sector son simples ejecutores de lo decidido por estos. Hay que establecer si puede hablarse del marketing como sistema de dirección en estas condiciones del sector estatal del turismo en Cuba, al menos a nivel de Destino Cuba. La misma situación se aplica para los elementos o variables del Mix, en particular la Distribución Comercial.

\section{Resultados}

\section{Resultados preliminares sobre distribución comercial del turismo en Cuba.}

Son escasos y poco precisos los datos sobre cómo se distribuye el producto cubano en los mercados que emiten hacia nuestro destino turístico. Un estudio en el Destino La Habana, dirigido por el que escribe, concluye que:

"Los indicadores no son suficientes para determinar las causas de las problemáticas existentes y no permiten examinar a fondo la distribución como proceso"y “(...) "no permiten la observación de este componente, pues no están orientados al análisis del mismo“(Errasti, Diaz. 2016)

No obstante, el análisis practicado a la información que pudo obtenerse en dicha investigación dio resultados que apuntan a la necesidad de perfeccionar la distribución comercial del producto turístico cubano. Los datos de los hoteles de la capital en 2015 dicen que sólo el $13 \%$ de las ventas son directas y el $87 \%$ restante se comercializa a través de intermediarios, tanto turoperadores como agencias de viajes. (Errasti, Diaz. 2016). Hay que tener en cuenta que se trata de un destino de ciudad donde se supone la turoperación debe tener menos peso.

$\mathrm{Al}$ analizar los ingresos se revela la notable diferencia entre el canal directo con ingresos promedio por pax de \$ 63 usd, un $27 \%$ superior al intermediado que fue sólo de \$ 50 usd. (Errasti, Diaz., 2016) 
Un resultado aún más preocupante encontramos al investigar la oferta hotelera de Sancti Spiritus que vende el $99 \%$ de su producto a través de intermediarios con precios más del $20 \%$ por debajo de lo que ingresaría por via directa. (Rodríguez, Díaz., 2016). Se trata de un tema no estudiado y poco abordado en el sector, el costo de la distribución turística., no obstante ser una de las principales preocupaciones y ocupación de las cadenas hoteleras presentes en el país. (Hosteltur, 04 JUNIO, 2015)

No hay encuestas a pie de escalerilla dirigidas a estudiar la distribución comercial. Se aplican encuestas para saber cómo llegaron a Cuba los que nos visitan, si por su cuenta o a través de una agencia de viajes y como se informaron sobre el destino. (Errasti, Diaz., 2016)

El caso del mercado potencial más importante para esta isla del Caribe, el de EE.UU., no es distinto en cuanto al conocimiento de cómo se distribuye el producto en ese país, aunque hay estudios enjundiosos, dirigidos por el que subscribe, sobre los segmentos identificados en este mercado que abordan el tema. (Turín y Díaz, 2011), (Ricardo, Díaz, 2013), (Castañeda, 2011),

Otro problema estudiado son las inconsistencias conceptuales sobre Distribución Comercial, Intermediación Comercial, los Actores que en ella intervienen y el papel que cada uno juega. (Díaz, A. 2012, 2016 y 2019). A esto se agrega la mistificación y distorsión del verdadero papel de las llamadas Tecnologías de Información y Comunicación, TIC, ya no tan nuevas. (Díaz, A. 2016) (Kotler, 1996)

Las cadenas hoteleras internacionales que operan en Cuba juegan un papel determinante en la comercialización de sus productos lo que rompe con la situación de monopolio del sector explicado anteriormente. Una condición de estas cadenas para operar en Cuba es la de garantizar el mercado para los hoteles que manejan por lo que están a cargo de la labor de marketing y la distribución comercial del producto que manejan.

Un estudio reciente sobre la Distribución Comercial del Hotel Parque Central, en contrato de Administración por Iberostar, deja claro que todo el marketing del producto queda en manos de la cadena extranjera y que, en cuanto a la distribución la Cadena Cubana propietaria del hotel solo tiene acceso limitado a dicha información. (Quintana, Díaz 2019)

Estas cadenas utilizan tres canales fundamentales: turoperadores, agencias de viajes, incluidas las OTAs y el canal directo a través sobre todo de sus sitios web para ventas online. El estudio mencionado revela que el peso principal recae entre turoperadores extranjeros y Receptivos cubanos, pero la tendencia de crecimiento se concentra en el canal directo a través del sitio web de la cadena extranjera. (Quintana, Díaz 2019)

Por el contrario las Cadenas Hoteleras nacionales apenas participan en la distribución de sus productos. Un estudio sobre el tema de este año en el Grupo Hotelero Gran Caribe, revela la limitada atención que se le presta a la distribución comercial, debido, primero a la centralización de la aplicación de esta variable en el MINTUR y segundo por descansar 
en las cadenas extranjeras con las que contratan la administración de buena parte de sus hoteles para la distribución comercial de los mismos. (Cepero, Díaz, 2019)

La distribución de los hoteles administrados directamente por el Grupo Gran Caribe, conocidos como "marca propia", se concentra en turoperadores y agencias de viajes online, OTAs, pues la vía on-line propia es mínima como se explica a continuación:

“Al respecto de la distribución online está instituido como política del MINTUR llevar a cabo estrategias que aprovechen las facilidades del entorno virtual, sin embargo, aún no está concebido este sistema a plenitud. Los hoteles apenas tienen ventas directas individualmente de forma online por medio de un sitio web propio, puesto que de los 19 hoteles Marca Propia que posee el Grupo Hotelero solo 4 cuentan con sitio web propio“. (Cepero, Díaz, 2019, 37)

Hay que agregar que ninguno de estos 4 hoteles que tiene sitio web puede vender on-line, pues no cuentan con pasarela de pagos para realizar la venta directa. Además, lo establecido es lo siguiente:

“La distribución directa online tiene como premisa siempre proteger la turoperación por medio de precios que sean mayores que los ofrecidos a los turoperadores, y lo más cercano posible al precio público. Esta estrategia se debe a que, aunque esta vía genere mayores utilidades promedio, es mayor la utilidad percibida por el volumen de venta de la turoperación". (Cepero, Díaz, 2019, 37, 38)

En suma, se protege el canal de distribución que rinde menos dividendos económicos por venta o pax.

A esto se suma la centralización total del tema, pues es el sitio web www.cubatravel.com.cu, operado por el MINTUR, el único que puede realizar ventas online, por tener todas las condiciones creadas, léase pasarela de pagos para poder culminar las ventas. Es el mismo Ministerio el que vende on-line.

Tanto la investigación de Quintana y Díaz como la de Cepero y Díaz establecen que los ingresos son mayores por pasajero cuando se trata de ventas directas, siguiéndole en resultados las ventas por OTAs y por último las realizadas a través de Receptivos cubanos y los turoperadores extranjeros, por lo que la estructura de la distribución afecta la mejoría de los resultados económicos de la planta hotelera estatal.

Otro asunto es el sector privado que oferta servicios al turismo, que ha crecido y se ha consolidado en el alojamiento, un $25 \%$ del total de turistas extranjeros al cierre del 2017 , y la restauración. (Rodríguez, Cuevas y Torres. 2017) Se trabaja en definir el impacto de las nuevas regulaciones recién publicadas para establecer en qué condiciones estas pequeñas empresas podrán utilizar el marketing en la gestión de las mismas. Más aún, hay que esperar como se aplicará la Constitución que finalmente nos hemos dado los cubanos y el marco legal que esta y las leyes que la complementen conformen como escenario para el funcionamiento del mercado nacional y su relación con el internacional 
y en base a esto, las posibilidades de una mayor aplicación del marketing como filosofía y sistema de dirección de todos los actores económicos del turismo en Cuba.

Hoy este incipiente y creciente sector privado utiliza todos los canales de distribución a su alcance, aunque con las limitaciones propias del país en cuanto a las vías on-line.

\section{Conclusiones}

Aunque la investigación sigue su curso, pueden llegarse a algunas conclusiones parciales:

- No está resuelto en nuestra economía el papel del mercado, que sigue marginado en la práctica.

- Ya se concibe al mercado como necesario en la dirección económica, incluso reconocido en la Constitución, pero continúa un centralismo reforzado con la creación de las llamadas OSDEs y la concentración en sólo dos decisores, el MINTUR y el Grupo GAVIOTA.

- $\quad$ Está seriamente limitada la aplicación del marketing a las empresas del turismo. No hay independencia económica suficiente para aplicarlo plenamente.

- La Distribución Comercial, no escapa a esta situación. Las cadenas extranjeras tienen independencia total al respecto, con limitaciones de precio mínimo establecido por el MINTUR. Las cadenas nacionales apenas participan en la distribución de sus productos y por tanto no aprenden ni se desarrollan sobre el tema.

- La distribución del producto hotelero cubano descansa en la turoperación en primer lugar, seguido de los Receptivos nacionales y las agencias de viajes extranjeras con una participación creciente de las OTAs y alrededor de sólo el $5 \%$ de ventas directas por las cadenas extranjeras y menos aún en el caso de las nacionales, lo que afecta los resultados económicos del turismo. Se trabaja en establecer el impacto de la quiebra del turoperador THOMAS COOK en el destino Cuba.

- $\quad$ Las agencias de viajes nacionales que gozaban de alguna independencia ahora están unificadas en el monopolio VIAJES CUBA, eliminando la competencia entre ellas y disminuyendo su participación en las decisiones sobre distribución y marketing.

- Los reiterados cambios en la legislación empresarial en los últimos 12 años no logran mejorar esta situación, por el contrario.

- Las decisiones de marketing están concentradas en el MINTUR. Más aún, este ministerio tiene incluso el sitio web para ventas directas on-line de los hoteles marca propia, algo así como el colmo de la centralización de la distribución comercial.

- Los contratos de administración con cadenas extranjeras no contribuyen a la adquisición del necesario "know how" sobre el tema, tan caro a nuestros ejecutivos de hoteles. 


\section{Referencias bibliográficas}

Arias Salazar, Claudio; Mendoza Tamayo, Nereyda, (2014, mayo agosto): "La comercialización de productos agropecuarios en Cuba. Un sistema a estudiar y perfeccionar". Universidad de Granma. Apartado 21. Bayamo. Granma. Cuba. Revista Granma Ciencia. Vol. 18, no. 2.

Arnaiz Sorí, Yudier; Pérez Pérez, Rigoberto; Aspiolea Ahu, María E., (2017, noviembre 30). “ANÁLISIS MARGINAL PARA LA DETERMINACIÓN DEL PRECIO ÓPTIMO EN EMPRESAS HOTELERAS DEL POLO TURÍSTICO JARDINES DEL REY EN LA PROVINCIA DE CIEGO DE ÁVILA, CUBA“, Universidad de Ciego de Ávila Máximo Gómez Báez. Vol.6, No. Especial UNICA.

Ayala Castro, Héctor, (2014, junio 30). "Evolución de Indicadores Turísticos de Cuba 2011-2013“; Retos Turísticos, Vol. 2 No. 2,

Ayala Castro, Héctor, (2016, enero-abril). "Un lustro de turismo receptor en Cuba: Tendencias de los ingresos turísticos”. Retos Turísticos, Vol. 15, No. 1, 2016.

Barreiro Pousa, Dr.C. Luis: (2013, abril). "MARKETING, RESPONSABILIDAD SOCIAL CORPORATIVA Y EMPRENDIMIENTO SOCIAL“ CPMARK, v. 1, n.1 (1) - Abril de 2013

Barreiro Pousa, Luis A. (2003) "Socialismo y Marketing", Publicado en la Revista ESPACIO (Cuba), Nro. 9.

Bartels, Robert (1976) “The History of Marketing Thought,” 2nd edition,

BORDEN, NEIL H. (1964); "The Concept of the Marketing Mix" Science in Marketing, George Schwartz (Ed.), New York: John Wiley,

Castañeda Quintero, Majel. (2011, 8 de junio): "Los Medios de Comunicación en Estados Unidos. Antecedentes, características y segmentación”. Tésis de Pregrado. Universidad de La Habana, Facultad de Turismo, Calle O N ${ }^{\circ} 262$, Vedado.

Cepero Peraza, Lianne y Díaz Medina, Antonio, (2019, mayo): “Propuesta de acciones para la mejora de la distribución comercial en los hoteles Marca Propia del Grupo Hotelero Gran Caribe*. Tésis de Pregrado. Universidad de La Habana, Facultad de Turismo, Calle $\mathrm{O}^{\circ}$ 262, Vedado.

Cuza Ceruto, José Francisco, (2013, junio): “Diagnóstico de la Gestión de la Calidad en el Establecimiento Marca Propia Hotel Playa Pesquero, perteneciente al Grupo Gaviota S.A". Tésis de Pregrado. Universidad de La Habana, Facultad de Turismo, Calle $\mathrm{O} \mathrm{N}^{\circ} 262$, Vedado. 
Decreto-ley no. 252. "sobre la continuidad y el fortalecimiento del sistema de dirección y gestión empresarial cubano" 2007.

Decreto no. 281. "reglamento para la implantacion y consolidacion del sistema de dirección y gestión empresarial estatal". Comité ejecutivo del consejo de ministros. 2007

Decreto-ley no. 320, "modificativo del decreto-ley no. 252 "sobre la continuidad y el fortalecimiento del sistema de dirección y gestión empresarial cubano”, 2014.

Decreto no. 323. "de la modificación del decreto no. 281, "reglamento para la implantación y consolidación del sistema de dirección y gestión empresarial estatal" 2014

Decreto no. 335/2017, “del sistema empresarial estatal cubano“. 2017.

Decreto no. 336/2017, "sistema de relaciones de las organizaciones superiores de dirección empresarial”. 2017

Del Castillo Sánchez, Luis, (2016, enero-junio): “El perfeccionamiento de la gestión de la empresa estatal y sus retos actuales en Cuba. Facultad de Economía, Universidad de La Habana. Economía y Desarrollo, vol. 156, núm. 1, pp. 142-154

Díaz Fernández, Dra. Ileana; Barreiro Pousa, Dr- Luis, (2006); "Marketing en Cuba, un estudio necesario", https://www.monografias.com/trabajos29/marketingcuba/marketing-cuba3.shtml

Díaz Fernández, Ileana - Hernández Ruiz, Alma - Barreiro Pousa, Luis A. (2009). "VALORACIONES SOBRE EL MARKETING EN CUBA". REDMARKA CIECID - Unidad de Investigación en Marketing Aplicado-Universidad de A Coruña Año I, Número 1, V1, pp.277-290

Díaz González y Pons García: "Necesidad de aplicación del marketing y la calidad percibida en la construcción del socialismo en Cuba" en Observatorio de la Economía Latinoamericana, $\mathrm{N}^{\mathrm{o}}$ 122, 2009. Texto completo en http//www.eumed.net/cursecon/ecolat/cu/2009/dgpg2.htm

Díaz Medina, Antonio, (2016, junio): "La llamada "Desintermediación" o el aumento de la productividad y explotación del trabajo en la comercialización de los viajes y el turismo. El caso de EE.UU.", TURyDES, Turismo y Desarrollo Local” (ISSN: 1988-5261),

Díaz Medina, Antonio (2012, diciembre): “Respuesta al Dr. Escalona a su “SOBRE EL ANÁLISIS MARXISTA DEL TURISMO“, TURyDES, Turismo y Desarrollo Local" (ISSN: 1988-5261) 
Díaz Medina, Antonio, (2012, diciembre): “Turismo y Conocimiento Científico, un primer acercamiento“. TURyDES, Turismo y Desarrollo Local” (ISSN: 19885261)

Errasti Hernández, Perla, Díaz Medina, Antonio, (2016): "Propuesta de un Sistema de Indicadores para la distribución del alojamiento hotelero estatal en La Habana“. Tésis de Pregrado. Universidad de La Habana, Facultad de Turismo, Calle O N ${ }^{\circ}$ 262, Vedado.

Excelencias Cuba, "Gaviota lidera cadenas hoteleras del Caribe y Latinoamérica": https://www.excelenciascuba.com/noticia/gaviota-lidera-cadenas-hoteleras-delcaribe-y-latinoamerica

Feinberg, Richard E. y Newfarmer, Richard S. (2016, diciembre): "Turismo en Cuba. En la ola hacia la prosperidad sostenible*, Latin America Initiative at Brookings • Kimberly Green Latin American and Caribbean Center.

Figueras, Dr. C. Miguel Alejandro, (2013). “Impactos del Turismo en la economía y sociedad cubana“, Miradas a la Economía Cubana, Editorial CAMINOS, Centro de Estudio de la Economia Cubana (CEEC), Universidad de La Habana

García Rabelo, Margarita, (2014, marzo 17): "Socialismo, mercado y desarrollo en el escenario cubano actual". Facultad de Economía, Universidad de La Habana, Cuba. Economía y Desarrollo. 152 (2). 20-36

Hernández Ruiz, Dra. Alma; Díaz Fernández, Dra. Ileana; Barreiro Pousa, Dr- Luis; s.f. “MARKETING EN CUBA: DÓNDE NOS ENCONTRAMOS". Centro de Estudios de Técnicas de Dirección (CETED). Facultad de Contabilidad y Finanzas. Universidad de La Habana.

Horta Coronado, Anet, Díaz Medina Antonio (2017, mayo): "Propuesta de estrategia de distribución del producto naturaleza de la Agencia de Viajes Gaviota Tours S.A.",. Tésis de Pregrado. Universidad de La Habana, Facultad de Turismo, Calle O N ${ }^{\circ}$ 262, Vedado.

Hosteltur, (2015, junio 4). “Meliá Hotels International vende a través de su página web el $37 \%$ del total". Recuperado de https://www.hosteltur.com/111390_melia-hotelsinternational-vende-traves-su-pagina-web-77-total.html

Jones, D.G. Brian, (2009 diciembre 18): “A History of Historical research in marketing" 03-Baker \& Saren-4011-CH-03:Baker \& Saren-4011-CH-03 7:27 PM Page 52

Kotler, Philip (2012): “Dirección de Marketing” Sexta Edición, Tomo II, pag 131

Laitamaki, Jukka M. (2018): "Sustainable Tourism in Cuban UNESCO World Heritage Sites: Focus on Historic City Centers", Jonathan M. Tisch Center for Hospitality 
and Tourism, New York University School of Professional Studies, New York City, USA

López Aguila, Yisel de la Caridad (2013): “Cuerpo de indicadores para el cálculo del nivel de servicio en hoteles 4 estrellas de ciudad del Grupo Cubanacan“. 2013. Tésis de Pregrado. Universidad de La Habana, Facultad de Turismo, Calle O N 262, Vedado.

Machado Chaviano, Esther Lidia; Hernández Aro, Yanet, (2013, enero). "Procedimiento participativo para el diseño de un producto turístico integrado en el destino Cuba". Alicante: Universidad de Alicante, 15 de, vol. 4, no 35, p. 69-92. [ISSN: 21731276]

Matín, Ramón, (2006): “El Comercio Internacional del Turismo en Condiciones de Oligopolio. El caso Cuba." Tesis presentada en opción al Grado Científico de Doctor en Ciencias Económicas.

Pérez Acevedo, Yamilé, (2016); "Propuesta de acciones para la comercialización electrónica del Hotel Telégrafo", Tésis de Pregrado. Universidad de La Habana, Facultad de Turismo, Calle $\mathrm{O}^{\circ}{ }^{\circ} 262$, Vedado.

Pons, Hugo M.; Utset, Laura: "LA PLANIFICACIÓN Y EL MARKETING COMO INSTRUMENTOS DE ADMINISTRACIÓN" Departamento de Ciencias Empresariales, Facultad de Economía, Universidad de la Habana. Recuperado de https://www.scribd.com/document/38785468/LA-PLANIFICACION-Y-ELMARKETING

Presentación del Ministro de Turismo. "DESARROLLO DEL TURISMO AL CIERRE DEL AÑO 2012, PERSPECTIVAS PARA EL 2013. IMPLEMENTACIÓN DE LOS LINEAMIENTOS".

Proyecto de Constitución de la Republica de Cuba, La Habana 2018.

Quintana Hernández, Adriana, Díaz Medina Antonio, (2019, mayo). "Identificación de los factores claves del éxito en la distribución del Grupo IBEROSTAR. Caso Hotel Iberostar Parque Central", Tésis de Pregrado. Universidad de La Habana, Facultad de Turismo, Calle $\mathrm{O} \mathrm{N}^{\circ} 262$, Vedado.

Resolución Económica del V Congreso del Partido Comunista de Cuba. La Habana, 810 de octubre de 1997. Ed. Política. La Habana.

Ricardo Díaz, Alejandro, Díaz Medina, Antonio, (2013, junio): "Exploraración de las potencialidades actuales para el segmento "People to People (P2P)" operados por la agencia Havanatur Celimar', Tésis de Pregrado. Universidad de La Habana, Facultad de Turismo, Calle $\mathrm{O} \mathrm{N}^{\circ} 262$, Vedado. 
Rodríguez Echemendía, Elizabeth y Díaz Medina, Antonio, (2016): ".COMPORTAMIENTO DE LOS CANALES DE DISTRIBUCIÓN DEL COMPLEJO HOTELERO RIJO-PLAZA-DON FLORENCIO“. Tésis de Pregrado. Universidad de La Habana, Facultad de Turismo, Calle O N $\mathrm{N}^{\circ}$ 262, Vedado.

Rodríguez García, Víctor; Villar López, Lydia, (2012, enero-junio), Turismo en Cuba: desempeño reciente y evaluación de la participación extranjera“: Economía y Desarrollo, vol. 147, núm. 1, , pp. 185-202

Rodríguez Gonzalez, Maite, (2017, septiembre-diciembre); “La gestión de la distribución en la agencia de viajes Cubatur S. A.", Retos Turísticos, Vol. 16, No. 3.

Rodríguez Noval, Raidel; Cuevas y Torres, (2017, junio): "Propuesta de acciones para la mejora de la distribución electrónica de alojamientos no estatales en el municipio Plaza de la Revolución”., Tésis de Pregrado. Universidad de La Habana, Facultad de Turismo, Calle $\mathrm{O} \mathrm{N}^{\circ} 262$, Vedado.

Seguí León, Gladys Caridad; Rodríguez Miló, Daymelis, (2012, diciembre). “EL MARKETING POR UNA MEJOR CALIDAD DE LOS SERVICIOS TURÍSTICOS EN CUBA“. Universidad de Pinar del Rio (Cuba). REDMARKA. Revista Digital de Marketing Aplicado

Shaw, Eric H.; Jones, D. G.Brian, (2005). A history of schools of marketing thought (2005) Vol 5, Marketing Theory, Issue 3,

Souto Anido, Lourdes; Pérez Herrera, Yaimara, (2012 julio-septiembre): "El rescate del enfoque de marketing en el contexto cubano actual“. Cofin Habana, n. ${ }^{\circ} 3$, , | pp. 30-35

Thomas“, Kitterlin-Lynch, Del Valle, (2015). “Cuba's Future Hospitality and Tourism Business: Opportunities and Obstacles“, Cornell University School of Hotel Administration, The Center for Hospitality Research (CHR)

Torres Hechavarría, M.Sc. Lisandra, (2017): "Propuesta de esquema metodológico para la evaluación de la sostenibilidad del desarrollo turístico de destinos. Caso La Habana“. Facultad de Turismo, Universidad de La Habana. La Habana : Editorial Universitaria, ISBN 978-959-16-3387-3.

TRAVEL \& TOURISM ECONOMIC IMPACT 2017, CUBA, World Travel \& Tourism Council (WTTC).

Turín López, Janet y Díaz Medina, Antonio (2011, junio): “Coherencia entre Cartera de productos de Havanatur Celimar y el comportamiento de compra de los CRE- 
EE.UU*. Tésis de Pregrado. Universidad de La Habana, Facultad de Turismo, Calle $\mathrm{O} \mathrm{N}^{\circ} 262$, Vedado.

Valdivia Barquin, Julio Antonio, (2010): "LA CRISIS ECONOMICA Y FINANCIERA MUNDIAL. SU IMPACTO EN EL TURISMO CUBANO“. Tésis de Pregrado. Universidad de La Habana, Facultad de Turismo, Calle O N ${ }^{\circ} 262$, Vedado.

\section{\Ciencia}




\section{PARA CITAR EL ARTÍCULO INDEXADO.}

Díaz Medina, A. (2021). Distribución comercial y marketing en el sector turístico cubano. Explorador Digital, 5(1), 44-63. https://doi.org/10.33262/exploradordigital.v5i1.1489

\section{Ciencia \\ LDigital}

El artículo que se publica es de exclusiva responsabilidad de los autores y no necesariamente reflejan el pensamiento de la Revista Explorador Digital.

El artículo queda en propiedad de la revista y, por tanto, su publicación parcial y/o total en otro medio tiene que ser autorizado por el director de la Revista Explorador Digital.
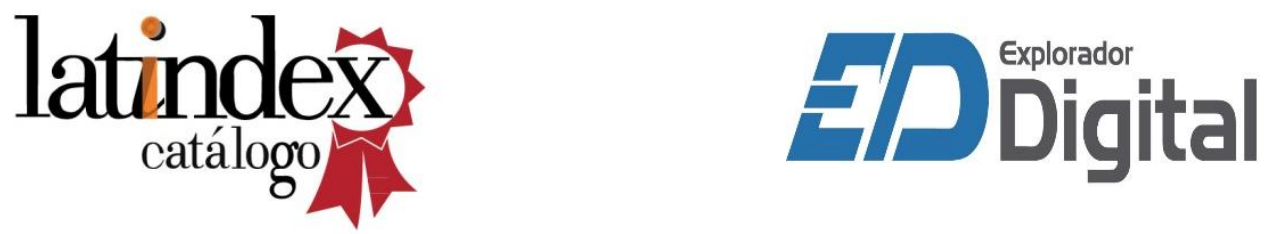